\title{
IMAGES IN ABDOMINAL TUBERCULOSIS
}

Dr. Gokul D. Yatheendranathan, Assistant Professor

\author{
Department of General Surgery, Shri Sathya Sai Medical College \& Research Institute, \\ Sri Balaji Vidyapeeth, Nellikuppam, Kancheepuram Dist., Tamil Nadu - 603 108, India.
}

Tuberculosis can involve any part of the gastrointestinal tract and is the sixth most frequent site of extra-pulmonary involvement after lymphatic, genitourinary, bone and joint, miliary and meningeal TB. Both the incidence and severity of abdominal tuberculosis is expected to increase with the increasing incidence of HIV infection. Tuberculosis bacteria reach the gastrointestinal tract via haematogenous spread, ingestion of infected sputum, or direct spread from infected contiguous lymph nodes and fallopian tubes. The gross pathology is characterized by transverse ulcers, fibrosis, thickening and strictures of the bowel wall, enlarged and matted mesenteric lymph nodes, omental thickening, and peritoneal tubercles.Peritoneal tuberculosis occurs in three forms: wet type with ascites, dry type with adhesions, and fibrotic type with omental thickening and loculated ascites.

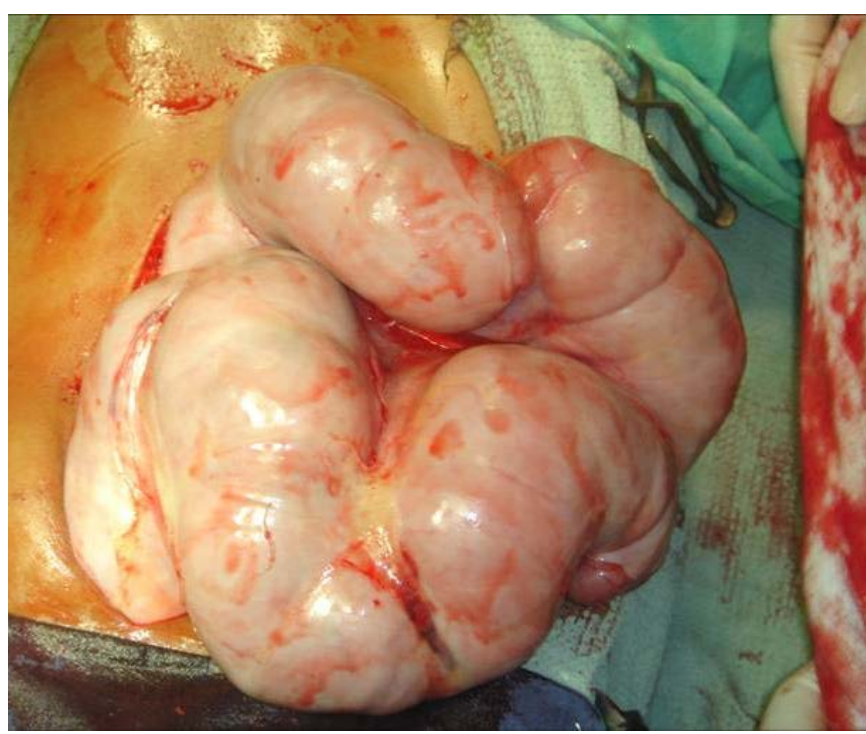

Fig 1: Tuberculous Intestinal cocoon
The most common site of involvement of the gastrointestinal tuberculosis is the ileocaecal region. Ileocaecal and small bowel tuberculosis presents with a palpable mass in the right lower quadrant and/or complications of obstruction, perforation or malabsorption especially in the presence of stricture.

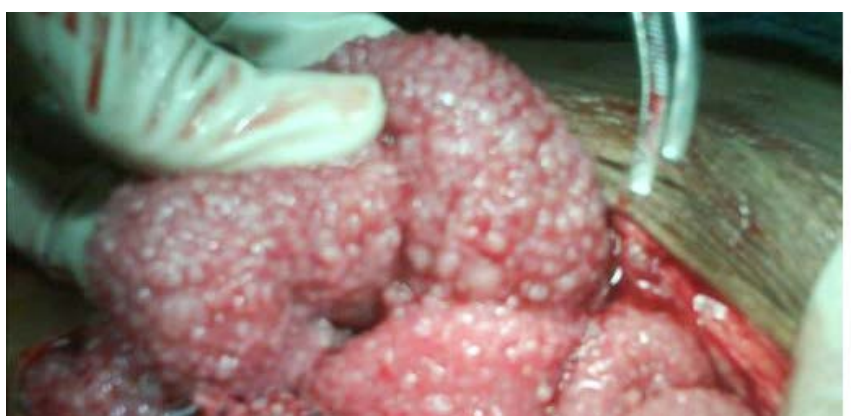

Fig 2: Illeal Stricture with proximal illeal perforation

Rare clinical presentations include dysphagia, odynophagia and a mid-oesophageal ulcer due to oesophageal tuberculosis, dyspepsia and gastric outlet obstruction due to gastroduodenal tuberculosis, lower abdominal pain and haematochezia due to colonic tuberculosis, and annular rectal stricture and multiple perianal fistulae due to rectal and anal involvement. Chest $\mathrm{X}$-rays show evidence of concomitant pulmonary lesions in less than 25 per cent of cases. Useful modalities for investigating a suspected case include small bowel barium meal, barium enema, ultrasonography, computed tomographic scan and colonoscopy. Ascitic fluid examination reveals straw coloured fluid with high protein, serum ascitic albumin gradient less than $1.1 \mathrm{~g} / \mathrm{dl}$, predominantly lymphocytic cells, and Adenosine Deaminase levels above 36 U/l. Laparoscopy is a very useful investigation in doubtful cases. Management 
is with conventional anti-tubercular therapy for at least 6 months. The recommended surgical procedures today are conservative and the period of preoperative drug therapy is controversial.

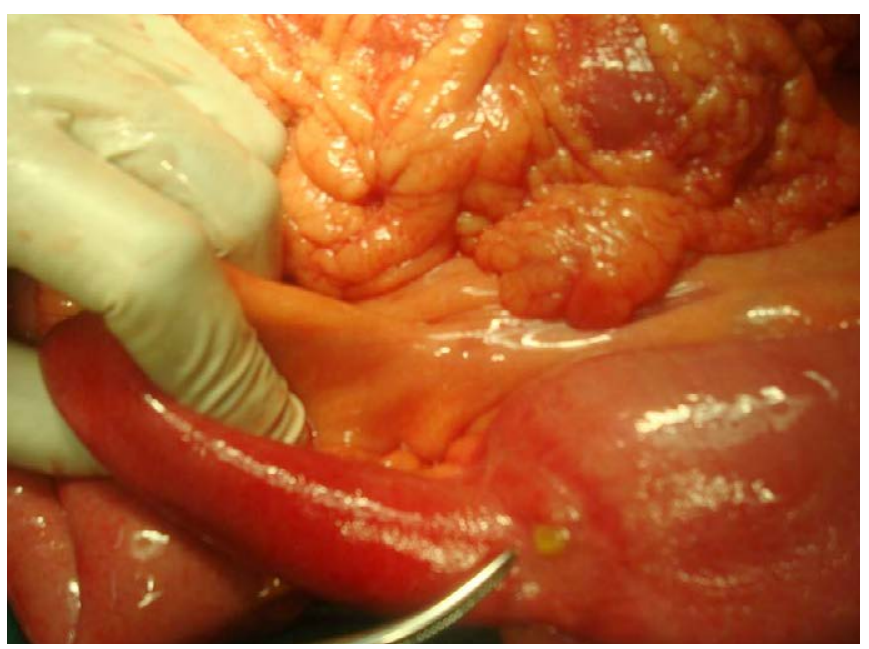

Fig 3: Peritoneal cavity \& small bowel studded with tubercles

\section{TAKE HOME POINTS}

1. Abdominal TB denotes involvement of GIT, peritoneum and/or draining lymph nodes.

2. Tubercular process can involve any organ in the abdominal cavity.

3. There is absence of extra abdominal lesions in the majority of patients.

4. A high index of clinical suspicion is necessary in any patient presenting with vague abdominal symptoms.

5. Radiological findings may be equivocal or merely contributory.

6. Most common site of involvement is ileo-caecal region.

7. A Histopathological confirmation is essential before making a firm diagnosis of tubercular abdomen

8. Diagnostic laparotomy or laparoscopy necessary for histological/microbiological diagnosis in peritoneal/ lymph node TB

9. Anti-tuberclous therapy forms the first line of management for patients with abdominal tuberculosis.

10. Therapeutic surgery essential for complications like Int. obstruction, perforation, peritonitis. 